Article

\title{
Synthesis, Anti-microbial and Molecular Docking Studies of Quinazolin-4(3H)-one Derivatives
}

\author{
Yahia Nasser Mabkhot ${ }^{1, *}$, Munirah S. Al-Har ${ }^{1,2}$, Assem Barakat ${ }^{1,3, *}$, Fahad D. Aldawsari ${ }^{1,4}$, \\ Ali Aldalbahi ${ }^{1}$ and Zaheer Ul-Haq ${ }^{5}$ \\ 1 Department of Chemistry, College of Science, King Saud University, P.O. Box 2455, \\ Riyadh 11451, Saudi Arabia; E-Mails: mun_eera2010@hotmail.com (M.S.A.-H.); \\ faldossri@kacst.edu.sa (F.D.A.); aaldalbahi@ksu.edu.sa (A.A.) \\ 2 Department of Chemistry, College of Sciences, Hail University, P.O. Box 2440, \\ Hail 81451, Saudi Arabia \\ 3 Department of Chemistry, Faculty of Science, Alexandria University, P.O. Box 426, \\ Ibrahimia 21321, Alexandria, Egypt \\ 4 King Abdulaziz City for Science and Technology, P.O. Box 6086, Riyadh-11442, Saudi Arabia \\ 5 Dr. Panjwani Center for Molecular Medicine and Drug Research, International Center for Chemical \\ and Biological Sciences, University of Karachi, Karachi-75210, Pakistan; \\ E-Mail: zaheer.qasmi@iccs.edu \\ * Authors to whom correspondence should be addressed; E-Mails: yahia@ksu.edu.sa (Y.N.M.); \\ ambarakat@ksu.edu.sa (A.B.); Tel.: +966-1467-5898 (Y.N.M.); +966-1467-5884 (A.B.); \\ Fax: +966-1467-5992 (Y.N.M. \& A.B.).
}

Received: 15 April 2014; in revised form: 6 June 2014 / Accepted: 9 June 2014 /

Published: 25 June 2014

\begin{abstract}
In this work, synthesis, antimicrobial activities and molecular docking studies of some new series of substituted quinazolinone $\mathbf{2} \mathbf{a}-\mathbf{h}$ and $\mathbf{3 a}-\mathbf{d}$ were described. Starting form 2-aminobenzamide derivatives $\mathbf{1}$, a new series of quinazolinone derivatives has been synthesized, in high yields, assisted by microwave and classical methods. Some of these substituted quinazolinones were tested for their antimicrobial activity against Gram-negative bacteria (Pseudomonas aeruginosa and Esherichia coli) and Gram-positive bacteria (Staphylococcus aureus, and Bacillus subtilis), and anti-fungal activity against (Aspergillus fumigatus, Saccharomyces cervevisiae, and Candida albicans) using agar well diffusion method. Among the prepared products, 3-benzyl-2-(4-chlorophenyl)quinazolin-4(3H)-one (3a) was found to exhibits the most potent in vitro anti-microbial activity with MICs of $25.6 \pm 0.5,24.3 \pm 0.4,30.1 \pm 0.6$, and $25.1 \pm 0.5 \mu \mathrm{g} / \mathrm{mL}$ against Staphylococcus aureus,
\end{abstract}


Bacillus subtilis, Pseudomonas aeruginosa and Esherichia coli, respectively. Compound 3a was found to exhibits the most potent in vitro anti-fungal activity with MICs of $18.3 \pm 0.6$, $23.1 \pm 0.4$, and $26.1 \pm 0.5 \mu \mathrm{g} / \mathrm{mL}$ against Aspergillus fumigatus, Saccharomyces cervevisiae, and Candidaal bicans, respectively.

Keywords: quinazolinone; antimicrobial agents; streptomycin; clotrimazole; molecular docking

\section{Introduction}

In the last decade, quinazolines have been extensively studied in medicinal chemistry. The quinazolinone scaffold is considered to be a motif structure. Quinazolinone ring system is found in a variety of bioactive natural as well as synthetic products. Many natural products contain quinazolinone core structures for example asperlicin C, sclerotigenin, circumdatin F, benzomalvin A, and many others have been documented as biologically important molecules [1-3]. Some synthetic quinazolinones, such as ispinesib, raltitrexed, halofuginone, tempostatin, etc. have been in the market or are currently in clinical trials for various cancer treatments. Quinazolines have exhibited therapeutic activities including antibacterial [4,5], antiviral [6], antifungal [7,8], antimalarial [9], antihypertensive [10], anticancer [11-13], diuretic [14,15], inhibition of derived growth factor receptor phosphorylation [16], antagonism of ghrelin receptor [17], anticonvulsant [18], COX-2 inhibitory activities [19,20] analgesic and anti-inflammatory.

Particularly, enzyme Sortase A involves in the pathogenesis of variety of bacterial infections, including respiratory tract, bloodstream, skin and tissue infection which serve to anchor some proteins responsible for virulence mainly by Gram +ve bacteria. Sortase A has been attracted great interest as potential drug targets since decades [21]. The inhibition of Sortase $A$ activity results in the separation of $S$. aureus from the host cells and ultimately alleviation of the infection. We used these newly synthesized active inhibitors to explore the binding cavity of Staphylococcus aureus Sortase A using GOLD docking program [21].

Recently, Mabkhot and co-workers have been involved in a research program aimed to development of new synthetic strategies for novel bioactive molecules and evaluation of their biological activity [22-28].

In this paper, we synthesized some new substituted quinazolinones as potential anti-microbial agents starting form 2-aminobenzamdie derivatives. The validity of this hypothesis was confirmed through preliminary in vitro anti-bacterial and anti-fungal screening of the desired molecules and their molecular docking studies. 


\section{Results and Discussion}

\subsection{Chemistry}

Synthesis of the desired compounds $\mathbf{2 a}-\mathbf{h}$ was achieved by allowing 2 -aminobenzamide derivatives $\mathbf{1 a}-\mathbf{h}[29,30]$ to undergo ring closure either with triethyl orthoformate under classical reflux condition as shown in Scheme 1. Alternatively, $\mathbf{2 a}-\mathbf{f}, \mathbf{h}$ can be obtained by microwave mediated methodology. It is proposed that the product $\mathbf{2} \mathbf{a}-\mathbf{h}$ was formed via initial nucleophilic addition of amide group into electrophilic carbon followed ring closure and elimination of three molecules of EtOH to give the desired products $\mathbf{2 a}-\mathbf{h}$. Assignment of structures of $\mathbf{2 a}-\mathbf{h}$ is based on elemental analysis and spectral data. Their IR spectra showed the disappearance of the characteristic absorption bands of $\mathrm{NH} \& \mathrm{NH}_{2}$ groups. Their ${ }^{1} \mathrm{H}-\mathrm{NMR}$ spectra showed in each case a characteristic singlet assigned to proton of $\mathrm{N}=\mathrm{CH}$. Their MS spectra are matched with the designed structures.

Scheme 1. Synthesis of quinazolin-4(3H)-one derivatives $\mathbf{2} \mathbf{a}-\mathbf{h}$ from 2-aminobenzamide derivatives $\mathbf{1 a}-\mathbf{h}$.<smiles>NC(=O)c1ccccc1N</smiles>

$1 \mathrm{a}-\mathrm{g}=\mathrm{H}$
$1 \mathrm{~h}=\mathrm{Cl}$<smiles>CCOC(OCC)OCC</smiles><smiles>COc1ccc(-n2cnc3ccccc3c2=O)cc1OC</smiles>

$2 \mathrm{~b}$<smiles>O=C(O)Cc1ccc(-n2cnc3ccccc3c2=O)cc1</smiles>

$2 \mathrm{~d}$<smiles>COc1ccc(-n2cnc3ccccc3c2=O)cc1</smiles>

$2 \mathrm{e}$<smiles>COc1cc(-n2cnc3ccccc3c2=O)cc(OC)c1OC</smiles>

$2 \mathrm{~g}$<smiles></smiles><smiles>COc1ccc(CCn2cnc3ccccc3c2=O)cc1OC</smiles>

2c<smiles>O=c1c2ccccc2ncn1Cc1ccc(Cl)cc1</smiles><smiles>COc1ccc(-n2cnc3c(Cl)cccc3c2=O)cc1</smiles>

$2 \mathrm{~h}$

Next, compounds 3a-d were synthesized by reaction of 2-aminobenzamide derivatives 1a-d with $p$-chlorobenzaldehyde, in DMF under reflux for $12 \mathrm{~h}$, as shown in Scheme 2. On the other hand, microwave mediated methodology gave the same 3a,b. It is assumed that the products 3a-d 
were formed via initial nucleophilic addition of amide to carbonyl group followed ring closure and elimination of $\mathrm{H}_{2} \mathrm{O}$ to give the desired products $\mathbf{3 a}-\mathbf{d}$. Elucidations of the chemical structures of $\mathbf{3 a}-\mathbf{d}$ are inferred from their spectroscopic and analytical data. Their IR spectra showed the disappearance of the characteristic absorption bands of $\mathrm{NH} \& \mathrm{NH}_{2}$ groups. The NMR and MS spectra are matched with the designed structures.

Scheme 2. Synthesis of quinazolin-4(3H)-one derivatives 3a-d from 2-aminobenzamide derivatives 1a-d.<smiles>[R]NC(=O)c1ccccc1N</smiles>

la-d

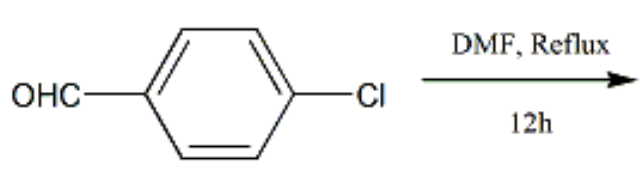<smiles>O=c1c2ccccc2nc(-c2ccc(Cl)cc2)n1Cc1ccccc1</smiles><smiles>COc1ccc(CCn2c(-c3ccc(Cl)cc3)nc3ccccc3c2=O)cc1OC</smiles><smiles>[R7]c1nc2ccccc2c(=O)n1-c1ccc(Cl)cc1</smiles>

b<smiles>O=C(O)Cc1ccc(-n2c(-c3ccc(Cl)cc3)nc3ccccc3c2=O)cc1</smiles>

\subsection{Antimicrobial Evaluation}

Antimicrobial activity of the synthesized compounds has been screened against micro-organisms representing Gram-(+ve) bacteria (Staphylococcus aureus and Bacillus subtilis), Gram-(-ve) bacteria (Escherichia coli and Pseudomonas aeruginosa), and fungi (Aspergillus fumigatus, Saccharomyces cerevisiae and Candida albicans), using the bioassay technique of antibiotics as antibacterial and 
antifungal standard drug specified in the US pharmacopeia at $25 \mu \mathrm{g} / \mathrm{mL}$. Among the screened compounds, only compound 3a showed a potent inhibitory effect against Gram- $(+\mathrm{ve})$ bacteria (Staphylococcus aureus and Bacillus subtilis) and Gram-(-ve) bacteria (Escherichia coli and Pseudomonas aeruginosa). Compound 3a found to be as similar potency as the standard drug (streptomycin). Nevertheless, 3a showed the strong inhibitory effect against fungi (Saccharomyces Cerevisiae, Aspergillus fumigatus and Candida albicans). The results indicate that compounds 3a has excellent biological activity and can be subjected for further evaluation for example enzyme inhibition. Clotrimazole and Streptomycin were used as standards [31]. The results obtained are listed in Table 1.

Table 1. Antimicrobial evaluation of the synthesized molecules.

\begin{tabular}{|c|c|c|c|c|c|c|c|}
\hline \multirow[b]{2}{*}{ Comp. No. } & \multicolumn{2}{|c|}{ Gram-Postive Bacteria } & \multicolumn{2}{|c|}{ Gram-Negative Bacteria } & \multicolumn{3}{|c|}{ Fungi } \\
\hline & $\begin{array}{c}\text { Staphylococcus } \\
\text { aureus }\end{array}$ & $\begin{array}{c}\text { Bacillus } \\
\text { subtilis }\end{array}$ & $\begin{array}{c}\text { Pseudomonas } \\
\text { aeruginosa }\end{array}$ & $\begin{array}{c}\text { Escherichia } \\
\text { coli }\end{array}$ & $\begin{array}{c}\text { Aspergillus } \\
\text { fumigatus }\end{array}$ & $\begin{array}{c}\text { Saccharomyces } \\
\text { cerevisiae }\end{array}$ & $\begin{array}{l}\text { Candida } \\
\text { albicans } \\
\end{array}$ \\
\hline $2 b$ & $7.8 \pm 0.4$ & $9.2 \pm 0.3$ & $9.7 \pm 0.3$ & $8.9 \pm 0.3$ & $9.5 \pm 0.4$ & $10.3 \pm 0.4$ & $10.5 \pm 0.4$ \\
\hline $2 c$ & $12.3 \pm 0.3$ & $13.1 \pm 0.4$ & $18.4 \pm 0.5$ & $15.2 \pm 0.4$ & $9.4 \pm 0.3$ & $15.2 \pm 0.4$ & $13.3 \pm 0.4$ \\
\hline 2d & $9.8 \pm 0.3$ & $12.2 \pm 0.2$ & $14.1 \pm 0.4$ & $13.8 \pm 0.3$ & $12.1 \pm 0.4$ & $13.7 \pm 0.3$ & $12.8 \pm 0.2$ \\
\hline $2 \mathrm{~g}$ & $10.3 \pm 0.2$ & $11.1 \pm 0.4$ & $10.9 \pm 0.4$ & $9.8 \pm 0.3$ & $11.3 \pm 0.5$ & $12.2 \pm 0.4$ & $11.4 \pm 0.3$ \\
\hline $2 h$ & $9.1 \pm 0.4$ & $9.6 \pm 0.3$ & $9.6 \pm 0.3$ & $9.8 \pm 0.3$ & $10.4 \pm 0.4$ & $11.3 \pm 0.4$ & $12.2 \pm 0.4$ \\
\hline $3 a$ & $25.6 \pm 0.5$ & $24.3 \pm 0.4$ & $30.1 \pm 0.6$ & $25.1 \pm 0.5$ & $18.3 \pm 0.6$ & $23.1 \pm 0.4$ & $26.1 \pm 0.5$ \\
\hline $\mathbf{3 b}$ & $10.4 \pm 0.3$ & $11.3 \pm 0.3$ & $11.1 \pm 0.2$ & $10.8 \pm 0.3$ & $9.8 \pm 0.2$ & $10.8 \pm 0.4$ & $10.3 \pm 0.5$ \\
\hline clotrimazole & & & & & $18.3 \pm 0.6$ & $23.1 \pm 0.4$ & $26.1 \pm 0.5$ \\
\hline streptomycin & $25.6 \pm 0.5$ & $24.3 \pm 0.4$ & $30.1 \pm 0.6$ & $25.1 \pm 0.5$ & & & \\
\hline
\end{tabular}

Inhibition zones $(\mathrm{mm})$.

\subsection{Molecular Docking Studies}

The antimicrobial potency of all the newly synthesized compounds were subjected for further docking studies to explore the binding pattern against methicillin resistant Staphylcoccus aureus (MRSA) [21]. PDB (ID 1T2W) [32] with $1.80 \AA$ resolution was retrieved from Brookhaven Protein Data Bank. From our previous studies residues including Ala92, Ala104, Glu105, Ala118, Pro163, Leu169, Gln172, Thr180, Ile182, Trp194 and Ile199 were found to be in the active site of the receptor, responsible for hydrophobic interactions. While Arg197 was the hotspot residue showing significant hydrogen bond within the binding pocket [21]. Conformational search of ligands were investigated via Gold docking program with extensive genetic algorithm. In this study, ten conformers were generated for each ligand using default parameters. Docking of all the newly synthesized inhibitors showed hydrogen bond interactions with Arg197 mentioned in Figure 1, supported our by previous findings [21].

The most active newly synthesized compound as an antimicrobial agent 3a showed potent inhibitory activity; involve in hydrogen bonding interaction with oxygen of quinazoline moiety at $2.13 \AA$ with Arg197. While the hydroxyl group of Ser116 showed strong hydrogen bond interaction with Ser116 at a bond distance of $2.29 \AA$ and chloro moiety of benzene ring attached to quinazoline showed hydrogen bond with Ala92 with a distance of 2.64 A. Hydrophobic interaction also observed between Trp194 and first benzene ring of the quinazoline moiety (Figure 2). 
Figure 1. Docked ligand at the receptor binding site. This picture represent 2D-interactions for the newly synthesized inhibitors within the binding pocket of target receptor using Poseview.
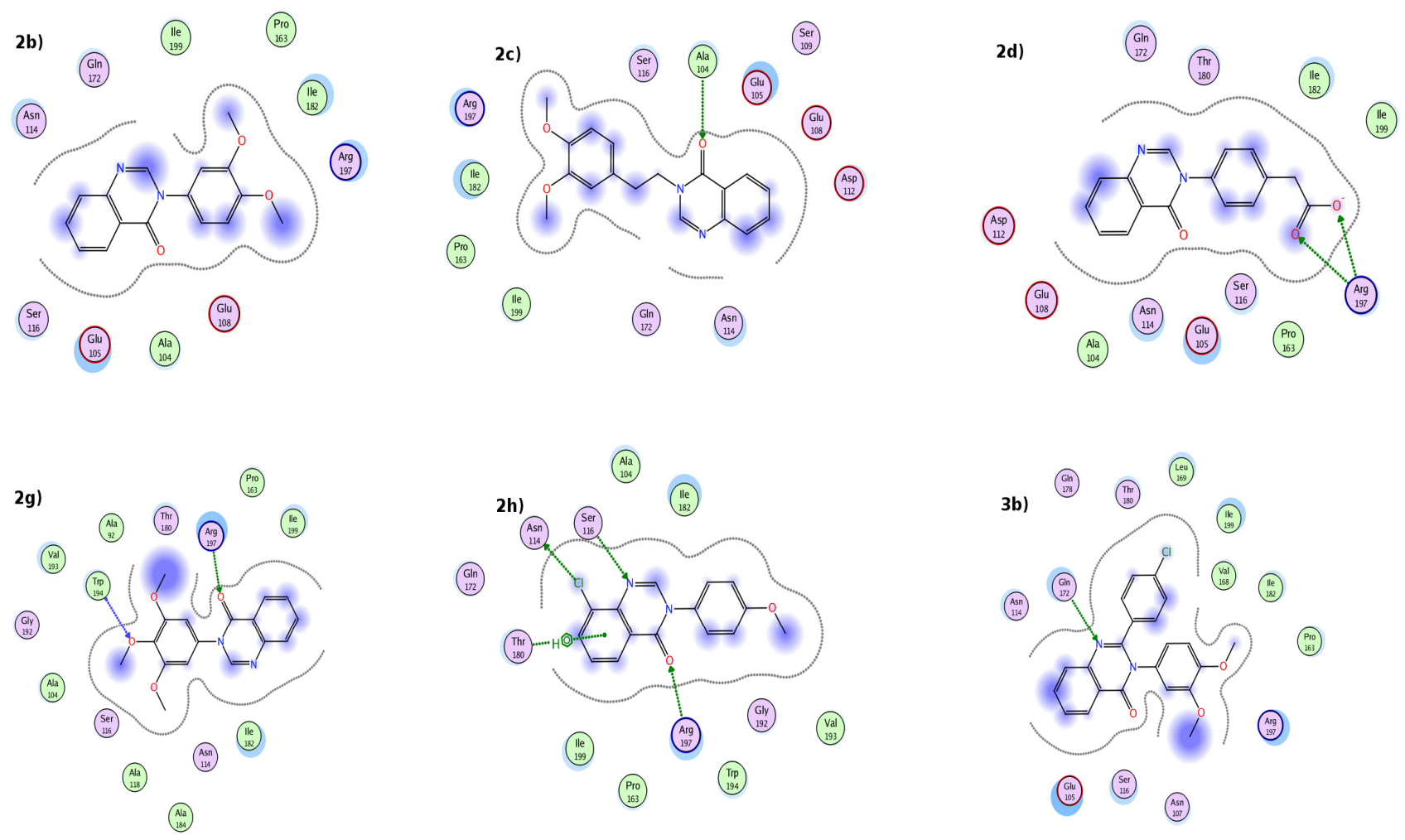

Figure 2. Molecular Docking Interaction diagram for the most potent compound 3a. Panel (a) is a two-dimensional representation of the docked pose by Poseview. Panel (b) representing three-dimensional view by MOE.

(2a)

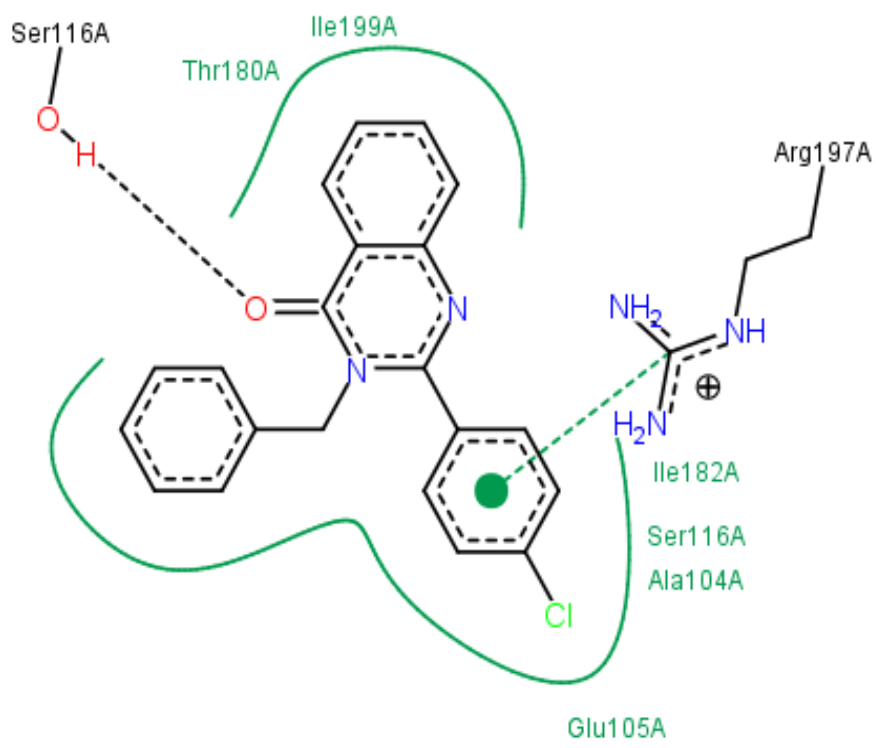


Figure 2. Cont.

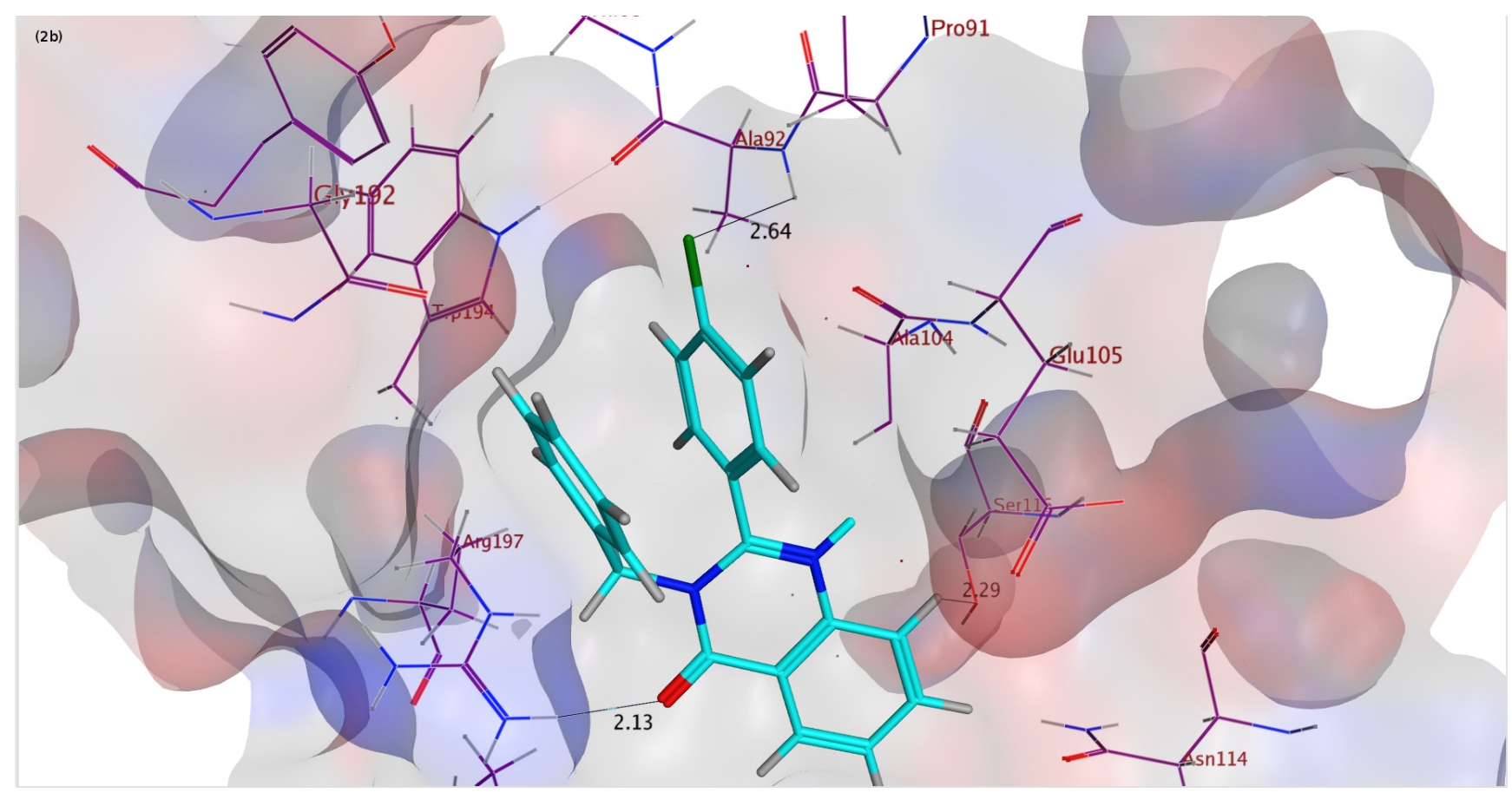

\section{Experimental Section}

General: The ${ }^{1} \mathrm{H}-\mathrm{NMR}$ and ${ }^{13} \mathrm{C}-\mathrm{NMR}$ spectra were run in DMSO- $d_{6}$ and recorded on a Varian Mercury or Jeol-400 NMR spectrometer. Coupling constants $J$ are given in Hz and chemical shifts $(\delta)$ are referred in ppm and related to that of the solvent. Abbreviations for multiplicity are as follows: $\mathrm{m}$ (multiplet), q (quadruplet), $\mathrm{t}$ (triplet), $\mathrm{d}$ (doublet) and $\mathrm{s}$ (singlet). IR spectra were recorded on a Perkin Elmer FT 1000 spectrophotometer using KBr pellets. Mass spectroscopy was measured on a Shimadzu GCMS-QP 1000 EX mass spectrometer at $70 \mathrm{eV}$. Melting points were measured on a Gallenkamp melting point apparatus in open glass capillaries and are uncorrected. Elemental analysis was carried out on an Elementar Vario EL analyzer.

\subsection{General Procedure for the Preparation of Qquinazolin-4(3H)-one Derivatives $\mathbf{2 a - h}$}

Method A (2a-h): To a mixture of 2-aminobenzamide derivatives 1a-h (2 mmol) was added triethyl orthoformate $(2-3 \mathrm{~mL})$ and the reaction mixture was heated under reflux for $12 \mathrm{~h}$. The reaction was monitored by TLC (EtOH: $\mathrm{CHCl}_{3}$ ), then left to cool to RT. The precipitated solid was filtered off, washed with diethyl ether and recrystallized from the proper solvent to afford the corresponding product $\mathbf{2 a}-\mathbf{h}$.

Method B (2a-f, h): A mixture of 2-aminobenzamide derivatives 1a-g, h (2 mmol) and triethyl orthoformate $(5-10 \mathrm{mmol})$ in the presence of view drops of DMF, was exposed to microwave irradiation $(140-420 \mathrm{w})$ for 4-10 min. The reaction mixture was left to cool to RT and methanol was added. The formed solid product was filtered off, washed with diethyl ether and recrystallized from the appropriate solvent to afford the corresponding product $\mathbf{2 a}-\mathbf{g}, \mathbf{h}$. 
3-Benzylquinazolin-4(3H)-one (2a). This compound was prepared from 2-amino- $N$-benzylbenzamide 1a according to method A or method B (4 min, $280 \mathrm{~W})$, and recyrtsallized from ethanol afford $2 \mathbf{a}$ as shining white needles; yield $\left(82^{\mathbf{a}}, 65^{\mathbf{b}_{\mathbf{0}}} \%\right.$ ); m.p. $116^{\circ} \mathrm{C}$; IR $v_{\max }(\mathrm{KBr}) 1630.75,1578.88 \mathrm{~cm}^{-1} ;{ }^{1} \mathrm{H}-\mathrm{NMR}$ $\left(400 \mathrm{MHz}, \mathrm{DMSO}-d_{6}\right)(\mathrm{ppm}): \delta 8.58(1 \mathrm{H}, \mathrm{s}), 8.16(1 \mathrm{H}, \mathrm{d}, J=8.0 \mathrm{~Hz}), 7.84(1 \mathrm{H}, \mathrm{t}, J=8.0 \mathrm{~Hz}), 7.70$ $(1 \mathrm{H}, \mathrm{d}, J=8.0 \mathrm{~Hz}), 7.55(1 \mathrm{H}, \mathrm{t}, J=8.0 \mathrm{~Hz}), 7.28-7.38(5 \mathrm{H}, \mathrm{m}), 5.20\left(2 \mathrm{H}, \mathrm{s}, \mathrm{CH}_{2}\right) ;{ }^{13} \mathrm{C}-\mathrm{NMR}: \delta 50.0$, 122.20, 126.68, 127.77, 127.83, 128.21(2C), 128.24, 129.21(2C), 135.00, 137.42, 148.48, 148.58,

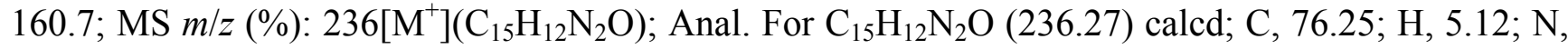
11.86; Found: C, 76.24; H, 5.12; N, 11.86.

3-(3,4-Dimethoxyphenyl)quinazolin-4(3H)-one (2b). Prepared from 2-amino- $N$-(3,4dimethoxyphenyl)benzamide (1b) according to method A or method B (6 min, $280 \mathrm{~W})$, and recyrstallized from ethanol to afford $\mathbf{2 b}$, dark powder; yield $\left(99^{\mathbf{a}}, 70^{\mathbf{b}_{\%}} \%\right)$; m.p. $140{ }^{\circ} \mathrm{C}$; IR $v_{\max }(\mathrm{KBr})$ 1669.14, $1595.03 \mathrm{~cm}^{-1} ;{ }^{1} \mathrm{H}-\mathrm{NMR}\left(400 \mathrm{MHz}, \mathrm{DMSO}-d_{6}\right)(\mathrm{ppm}): \delta 8.31(1 \mathrm{H}, \mathrm{s}), 8.20(1 \mathrm{H}, \mathrm{d}, J=8.0 \mathrm{~Hz})$, $7.88(1 \mathrm{H}, \mathrm{t}, J=8.0 \mathrm{~Hz}), 7.74(1 \mathrm{H}, \mathrm{d}, J=8.0 \mathrm{~Hz}), 7.60(1 \mathrm{H}, \mathrm{t}, J=8.0 \mathrm{~Hz}), 7.18(1 \mathrm{H}, \mathrm{s}), 7.1(1 \mathrm{H}, \mathrm{d}$, $J=8.0 \mathrm{~Hz}), 7.06(1 \mathrm{H}, \mathrm{d}, J=8.0 \mathrm{~Hz}), 3.77,3.83\left(\right.$ each $\left.3 \mathrm{H}, \mathrm{s}, 2\left(\mathrm{OCH}_{3}\right)\right) ;{ }^{13} \mathrm{C}-\mathrm{NMR}: \delta 58.0,112.13(2 \mathrm{C})$, $120.11,122.51,126.98,127.84,127.91,130.96,135.14,148.13,148.31,149.39,149.49$, 160.7; MS

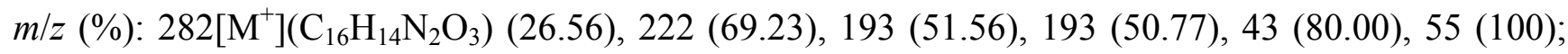
Anal. for $\mathrm{C}_{16} \mathrm{H}_{14} \mathrm{~N}_{2} \mathrm{O}_{3}$ (282.29) calcd; C, 68.07; H, 5.00; N, 9.92; Found: C, 68.10; H, 5.03; N, 9.91.

3-(3,4-Dimethoxyphenethyl)quinazolin-4(3H)-one (2c). Prepared from 2-amino- $N-(3,4-$ dimethoxyphenethyl)benzamide 1c according to method A, as beige powder; yield $\left(95^{\mathbf{a}_{0}} \%\right)$; m.p. $140{ }^{\circ} \mathrm{C}$; IR $v_{\max }(\mathrm{KBr}) 1681.65,1642.74 \mathrm{~cm}^{-1} ;{ }^{1} \mathrm{H}-\mathrm{NMR}\left(400 \mathrm{MHz}, \mathrm{DMSO}-d_{6}\right)(\mathrm{ppm}): \delta 8.76(1 \mathrm{H}, \mathrm{s}), 8.36(1 \mathrm{H}$, $\mathrm{d}, J=7.8 \mathrm{~Hz}), 7.65(1 \mathrm{H}, \mathrm{d}, J=7.8 \mathrm{~Hz}), 7.46(1 \mathrm{H}, \mathrm{t}, J=7.8 \mathrm{~Hz}), 7.12(1 \mathrm{H}, \mathrm{t}, J=7.8 \mathrm{~Hz}), 6.83-6.87(2 \mathrm{H}$, m), $6.75(1 \mathrm{H}, \mathrm{d}, J=8.0 \mathrm{~Hz}), 3.71\left(6 \mathrm{H}, \mathrm{s},\left(2\left(\mathrm{OCH}_{3}\right)\right), 3.47\left(2 \mathrm{H}, \mathrm{t}, \mathrm{CH}_{2}\right), 2.79\left(2 \mathrm{H}, \mathrm{t}, \mathrm{CH}_{2}\right) ;{ }^{13} \mathrm{C}-\mathrm{NMR}: \delta\right.$ 25.3, 34.9, 55.9, 56.0,112.37, 113.07, 120.93, 121.09 (2C), 121.65, 123.00, 128.47, 132.24, 132.32, 139.36, 147.78, 149.10, 168,7; MS $m / z(\%): 310[\mathrm{M}+]\left(\mathrm{C}_{18} \mathrm{H}_{18} \mathrm{~N}_{2} \mathrm{O}_{3}\right)$ (15.62), 293 (100), 275 (35.94), 28 (21.87), 44 (20.31), 69 (18.75); Anal. For $\mathrm{C}_{18} \mathrm{H}_{18} \mathrm{~N}_{2} \mathrm{O}_{3}$ (310.35) calcd; C, 69.66; H, 5.85; N, 9.03; Found: C, 69.64; H, 5.83; N, 9.05.

2-(4-(4-Oxoquinazolin-3(4H)-yl)phenyl)acetic Acid (2d). Compound 2d was prepared from 2-(4-(2aminobenzamido)phenyl)acetic acid 1d according to method A or method B (5 min, $280 \mathrm{~W})$, and recrystallized from ethanol afford $2 \mathbf{d}$ as, brownish powder; yield $\left(85^{\mathbf{a}}, 70^{\mathbf{b}_{\%}} \%\right.$ ); m.p. $267{ }^{\circ} \mathrm{C}$; IR $v_{\max }$ (KBr) 3678.56, 3449.51, 1687.32, 1611.24, $1564.26 \mathrm{~cm}^{-1}$; ${ }^{1} \mathrm{H}-\mathrm{NMR}$ (400 MHz, DMSO- $d_{6}$ ) (ppm): $\delta$ $8.35(1 \mathrm{H}, \mathrm{s}), 8.21(1 \mathrm{H}, \mathrm{d}, J=7.7 \mathrm{~Hz}), 7.89(1 \mathrm{H}, \mathrm{t}, J=7.7 \mathrm{~Hz}), 7.75(1 \mathrm{H}, \mathrm{d}, J=7.7 \mathrm{~Hz}), 7.60(1 \mathrm{H}, \mathrm{t}$, $J=7.7 \mathrm{~Hz}), 7.49(2 \mathrm{H}, \mathrm{d}, J=8.0 \mathrm{~Hz}), 7.45(2 \mathrm{H}, \mathrm{d}, J=8.0 \mathrm{~Hz}), 3.69\left(2 \mathrm{H}, \mathrm{s}, \mathrm{CH}_{2}\right) ;{ }^{13} \mathrm{C}-\mathrm{NMR}: \delta 25.3$, 122.46, 127.01, 127.79 (2C), 127.89, 127.98, 130.81(2C), 135.23, 136.35, 136.63, 147.78, 148.29, 160.6, 173.1; $\mathrm{MS} \mathrm{m} / z$ (\%): $280\left[\mathrm{M}^{+}\right]\left(\mathrm{C}_{16} \mathrm{H}_{12} \mathrm{~N}_{2} \mathrm{O}_{3}\right)$ (100), 250 (19.05), 235 (92.55), 207 (15.24),129 (32.38), 107 (56.19); Anal. for $\mathrm{C}_{16} \mathrm{H}_{12} \mathrm{~N}_{2} \mathrm{O}_{3}$ (280.28) calcd; C, 68.56; H, 4.32; N, 9.99; Found: C, $68.55 ; \mathrm{H}, 4.31 ; \mathrm{N}, 10.01$.

3-(4-Methoxyphenyl)quinazolin-4(3H)-one (2e). Compound 2e was prepared from 2-amino- $N$-(4methoxyphenyl)benzamide 1e according to method A or method B (4 min,420 W), and recrystallized from ethanol afford $2 \mathbf{e}$ as shining white needles; yield $\left(99^{\mathbf{a}}, 95^{\mathbf{b}_{\%}}\right)$; m.p. $195{ }^{\circ} \mathrm{C}$; IR $v_{\max }(\mathrm{KBr})$ 
1681.54, $1610.01 \mathrm{~cm}^{-1} ;{ }^{1} \mathrm{H}-\mathrm{NMR}\left(400 \mathrm{MHz}, \mathrm{DMSO}-d_{6}\right)(\mathrm{ppm}): \delta 8.31(1 \mathrm{H}, \mathrm{s}), 8.20(1 \mathrm{H}, \mathrm{d}, J=8.0 \mathrm{~Hz})$, $8.20(1 \mathrm{H}, \mathrm{d}, J=8.0 \mathrm{~Hz}), 7.88(1 \mathrm{H}, \mathrm{t}, J=8.0 \mathrm{~Hz}), 7.74(1 \mathrm{H}, \mathrm{d}, J=8.0 \mathrm{~Hz}), 7.60(1 \mathrm{H}, \mathrm{t}, J=8.0 \mathrm{~Hz}), 7.46$ $(2 \mathrm{H}, \mathrm{d}, J=8.0 \mathrm{~Hz}), 7.10(2 \mathrm{H}, \mathrm{d}, J=8.0 \mathrm{~Hz}), 3.83\left(3 \mathrm{H}, \mathrm{s}, \mathrm{OCH}_{3}\right) ;{ }^{13} \mathrm{C}-\mathrm{NMR}: \delta 56.1,114.90(2 \mathrm{C})$, $122.49,126.98,127.85,127.91,129.24$ (2C), 130.86, 135.14, 148.07, 148.34, 159.82, 160.8; MS m/z (\%): $252\left[\mathrm{M}^{+}\right]\left(\mathrm{C}_{15} \mathrm{H}_{12} \mathrm{~N}_{2} \mathrm{O}_{2}\right)$ (100), $253\left[\mathrm{M}^{+}+1\right]$ (20.80), $254\left[\mathrm{M}^{+}+2\right]$ (2.20), 237 (18.30), 209 (10.92), 129 (18.44); Anal. for $\mathrm{C}_{15} \mathrm{H}_{12} \mathrm{~N}_{2} \mathrm{O}_{2}$ (252.27) calcd; C, 67.82; H, 4.82; N, 12.17. Found: C, 67.81; H, $4.80 ; \mathrm{N}, 12.25$.

3-(4-Chlorobenzyl)quinazolin-4(3H)-one (2f). Compound 2f was prepared from 2-amino- $N$-(4chlorobenzyl)benzamide $\mathbf{1 f}$ according to method A or method B (2 min, $280 \mathrm{~W})$ and recrystallized from methanol afford $2 \mathbf{f}$ as white powder; yield $\left(88^{\mathbf{a}}, 62^{\mathbf{b}_{\%}} \%\right)$; m.p. $155^{\circ} \mathrm{C}$; IR $v_{\max }(\mathrm{KBr}) 1602.24$, $1531.10 \mathrm{~cm}^{-1} ;{ }^{1} \mathrm{H}-\mathrm{NMR}\left(400 \mathrm{MHz}, \mathrm{DMSO}-d_{6}\right)(\mathrm{ppm}): \delta 8.60(1 \mathrm{H}, \mathrm{s}), 8.15(1 \mathrm{H}, \mathrm{d}, J=8.0 \mathrm{~Hz}), 7.83$ $(1 \mathrm{H}, \mathrm{t}, J=8.0 \mathrm{~Hz}), 7.70(1 \mathrm{H}, \mathrm{d}, J=8.0 \mathrm{~Hz}), 7.31-7.41(4 \mathrm{H}, \mathrm{m}), 6.56(1 \mathrm{H}, \mathrm{t}, J=8.0 \mathrm{~Hz}), 5.19(2 \mathrm{H}, \mathrm{s}$, $\left.\mathrm{CH}_{2}\right)$; ${ }^{13} \mathrm{C}-\mathrm{NMR}: \delta 49.9,126.66,127.82,128.74,150.10,129.15(2 \mathrm{C}), 129.57,130.23(2 \mathrm{C}), 132.40$, 132.88, 135.07, 136.40, 148.5; MS $m / z(\%): 270\left[\mathrm{M}^{+}\right]\left(\mathrm{C}_{15} \mathrm{H}_{11} \mathrm{~N}_{2} \mathrm{OCl}\right)$; Anal. for $\mathrm{C}_{15} \mathrm{H}_{11} \mathrm{ClN}_{2} \mathrm{O}(270.71)$ calcd; C, 66.55; H, 4.10; Cl, 13.10; N, 10.35; Found: C, 66.54; H, 4.11; Cl, 13.09; N, 10.33.

3-(3,4,5-Trimethoxyphenyl)quinazolin-4(3H)-one (2g). Compound 2g was prepared from 2-amino- $N$ (3,4,5-trimethoxyphenyl)benzamide $1 \mathrm{~g}$ according to method A or method B (4 min,280 W), as brownish powder; yield $\left(98^{\mathbf{a}}, 88^{\mathbf{b}_{\mathbf{b}}}\right.$ ); m.p. $115^{\circ} \mathrm{C}$; IR $v_{\max }(\mathrm{KBr}) 1680.00,1607.17 \mathrm{~cm}^{-1}$; ${ }^{1} \mathrm{H}-\mathrm{NMR}(400$ MHz, DMSO- $\left.d_{6}\right)(\mathrm{ppm}): \delta 8.34(1 \mathrm{H}, \mathrm{s}), 8.21(1 \mathrm{H}, \mathrm{d}, J=8.0 \mathrm{~Hz}), 7.88(1 \mathrm{H}, \mathrm{t}, J=8.0 \mathrm{~Hz}), 7.75(1 \mathrm{H}, \mathrm{d}$, $J=8.0 \mathrm{~Hz}), 7.60(1 \mathrm{H}, \mathrm{t}, J=8.0 \mathrm{~Hz}), 7.17(1 \mathrm{H}, \mathrm{s}), 6.92(1 \mathrm{H}, \mathrm{s}), 3.77,3.79\left(\right.$ each $\left.3 \mathrm{H}, \mathrm{s}, 2\left(\mathrm{OCH}_{3}\right)\right), 3.76$ $\left(3 \mathrm{H}, \mathrm{s}, \mathrm{OCH}_{3}\right) ;{ }^{13} \mathrm{C}-\mathrm{NMR}: \delta 56.3,56.7,106.11(2 \mathrm{C}), 122.49,126.97,127.84,127.95,133.86,135.20$, 138.07, 148.26, 153.09, 153.59, 160.6; MS m/z (\%): 312[ $\left.\mathrm{M}^{+}\right]\left(\mathrm{C}_{17} \mathrm{H}_{16} \mathrm{~N}_{2} \mathrm{O}_{4}\right)$ (6.25), 297 (31.25), 194 (100), 165 (25.00), 87 (17.18), 68 (23.44); Anal. for $\mathrm{C}_{17} \mathrm{H}_{16} \mathrm{~N}_{2} \mathrm{O}_{4}$ (312.32) calcd; C, 65.38; H, 5.16; N, 8.97; Found: C, 65.39; H, 5.15; N, 8.93.

8-Chloro-3-(4-methoxyphenyl)quinazolin-4(3H)-one (2h). $2 \mathbf{h}$ was prepared from 2-amino-3-chloro- $N$ (4-methoxyphenyl)benzamide $\mathbf{1 h}$ according to method A, or method $\mathrm{B}(4 \mathrm{~min}, 280 \mathrm{~W})$ as shining beige powder; yield $\left(97^{\mathbf{a}}, 60^{\mathbf{b}_{0}}\right.$ ); m.p. $175^{\circ} \mathrm{C}$; IR $v_{\max }(\mathrm{KBr}) 1631.68,1610.34 \mathrm{~cm}^{-1} ;{ }^{1} \mathrm{H}-\mathrm{NMR}(400 \mathrm{MHz}$, DMSO- $\left.d_{6}\right)(\mathrm{ppm}): \delta 8.43(1 \mathrm{H}, \mathrm{s}), 7.57-7.62(3 \mathrm{H}, \mathrm{m}), 7.43(1 \mathrm{H}, \mathrm{d}, J=8.0 \mathrm{~Hz}), 6.92(2 \mathrm{H}, \mathrm{d}, J=8.8 \mathrm{~Hz})$, $6.66(1 \mathrm{H}, \mathrm{t}, J=8.0 \mathrm{~Hz}), 3.74\left(3 \mathrm{H}, \mathrm{s}, \mathrm{OCH}_{3}\right) ;{ }^{13} \mathrm{C}-\mathrm{NMR}: \delta 55.74,114.26(2 \mathrm{C}), 114.94,115.90,118.17$, $119.48, \quad 122.88$ (2C), 128.21, 129.21, 132.42, 145.53, 156.21, 167.30; $\mathrm{MS} \quad \mathrm{m} / z \quad(\%)$ : $28\left[\mathrm{M}^{+}\right]\left(\mathrm{C}_{15} \mathrm{H}_{11} \mathrm{~N}_{2} \mathrm{O}_{2} \mathrm{Cl}\right)$ (61.50), $288\left[\mathrm{M}^{+}+2\right]\left(\mathrm{C}_{15} \mathrm{H}_{11} \mathrm{~N}_{2} \mathrm{O}_{2} \mathrm{Cl}\right)$ (21.02), 187 (100), 244 (50.94), 43 (39.00), 189 (33.10); Anal. for $\mathrm{C}_{15} \mathrm{H}_{11} \mathrm{ClN}_{2} \mathrm{O}_{2}$ (286.71) calcd; C, 62.84; H, 3.87; Cl, 12.37; N, 9.77; Found: C, 62.85; H, 3.87; Cl, 12.36; N, 9.78.

\subsection{General Method for Preparation of Compounds Derivatives 3a-0}

Method A (3a-d): To a solution of 2-aminobenzamide derivatives 1a-d (2 mmol) in DMF (2-3 mL), and $p$-chlorobenzaldehyde $(2 \mathrm{mmol})$ was added. The reaction mixture was heated under reflux for $12 \mathrm{~h}$. The reaction mixture was monitored by $\mathrm{TLC}\left(\mathrm{EtOH} / \mathrm{CHCl}_{3}\right)$, then left to cool to RT, and poured 
into cold water $(50 \mathrm{~mL})$, the precipitated solid product was filtered off, and dried to afford the corresponding product $\mathbf{3 a}-\mathbf{d}$.

Method B (3a,b): A mixture of 2-aminobenzamide derivatives 1a,b (2 mmol) and $p$-chlorobenzaldehyde $(2 \mathrm{mmol})$ in the presence of view drops from DMF, was exposed to microwave irradiation (420-560 w) for 5-10 min. The reaction mixture was left to cool to RT. Water was added and the formed solid product was filtered off afforded the corresponding product $\mathbf{3 a}, \mathbf{b}$.

3-Benzyl-2-(4-chlorophenyl)quinazolin-4(3H)-one (3a). 3a was prepared from 2-amino- $N$ benzylbenzamide 1a according to method A, or method B (5 min, $420 \mathrm{~W})$ as white scales; yield $\left(82^{\mathrm{a}}\right.$, $63^{\mathbf{b}_{\mathbf{0}}}$ ); m.p. $101{ }^{\circ} \mathrm{C}$; IR $v_{\max }(\mathrm{KBr}) 1617.23,1589.98 \mathrm{~cm}^{-1}$; ${ }^{1} \mathrm{H}-\mathrm{NMR}$ (400 MHz, DMSO- $\left.d_{6}\right)(\mathrm{ppm}): \delta$ $7.85(1 \mathrm{H}, \mathrm{d}, J=8.0 \mathrm{~Hz}), 7.79(2 \mathrm{H}, \mathrm{d}, J=8.8 \mathrm{~Hz}), 7.51-7.56(3 \mathrm{H}, \mathrm{m}), 7.25-7.36(7 \mathrm{H}, \mathrm{m}), 4.49(2 \mathrm{H}, \mathrm{s}$, $\left.\mathrm{CH}_{2}\right) ;{ }^{13} \mathrm{C}-\mathrm{NMR}: \delta$ 40.3, 119.76, 126.81, 127.45 (2C), 128.02 (2C), 128.89 (2C), 129.52 (2C), 129.98, 131.14 (2C), 132.16, 134.84, 137.12, 139.61, 149.24, 161.74, 166.5; $\mathrm{MS} \mathrm{m} / \mathrm{z}(\%): 346\left[\mathrm{M}^{+}\right]$ $\left(\mathrm{C}_{21} \mathrm{H}_{15} \mathrm{~N}_{2} \mathrm{OCl}\right)(100 \%), 347\left[\mathrm{M}^{+}+1\right]$ (23.00), 348[M+2] (33.61), $349\left[\mathrm{M}^{+}+3\right](6.90 \%), 155(60.55 \%)$, 91 (78.03\%); Anal. for $\mathrm{C}_{21} \mathrm{H}_{15} \mathrm{ClN}_{2} \mathrm{O}$ (346.81) calcd; C, 72.73; H, 4.36; Cl, 10.22; N, 8.08; Found: C, $72.75 ; \mathrm{H}, 4.36 ; \mathrm{Cl}, 10.23 ; \mathrm{N}, 8.10$.

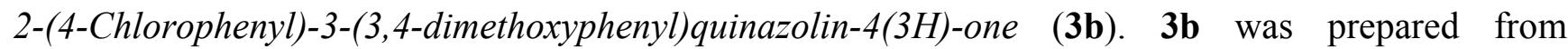
2-amino- $N$-(3,4-dimethoxyphenyl)benzamide $\mathbf{1 b}$ according to method A, or method B (5 min, $560 \mathrm{~W}$ ) as shining pale green scales; yield $\left(87^{\mathbf{a}}, 77^{\mathbf{b}_{0}} \%\right)$; m.p. $147^{\circ} \mathrm{C}$; IR $v_{\max }(\mathrm{KBr}) 1615.17,1561.63 \mathrm{~cm}^{-1}$; ${ }^{1} \mathrm{H}-\mathrm{NMR}\left(400 \mathrm{MHz}, \mathrm{DMSO}-d_{6}\right)(\mathrm{ppm}): \delta 8.04(2 \mathrm{H}, \mathrm{d}, J=8.0 \mathrm{~Hz}), 7.92(1 \mathrm{H}, \mathrm{d}, J=8.0 \mathrm{~Hz}), 7.60-7.65$ $(3 \mathrm{H}, \mathrm{m}), 7.38-7.41(3 \mathrm{H}, \mathrm{m}), 7.33(1 \mathrm{H}, \mathrm{d}, J=8.0 \mathrm{~Hz}), 6.91(1 \mathrm{H}, \mathrm{t}, J=8.0 \mathrm{~Hz}), 3.70,3.72($ each $3 \mathrm{H}, \mathrm{s}$, $\left(2\left(\mathrm{OCH}_{3}\right)\right) ;{ }^{13} \mathrm{C}-\mathrm{NMR}: \delta 55.8,56.3,111.95,112.61,119.89,126.99,129.60(2 \mathrm{C}), 129.77,130.09$, 131.22 (2C), 132.55, 132.89, 134.97, 137.42, 145.62, 149.11,149.20, 162.04, 164.6; MS m/z (\%): 392 $\left[\mathrm{M}^{+}\right]\left(\mathrm{C}_{22} \mathrm{H}_{17} \mathrm{~N}_{2} \mathrm{O}_{3} \mathrm{Cl}\right)(20.90 \%), 393\left[\mathrm{M}^{+}+1\right](5.34), 391\left[\mathrm{M}^{+}-1 \mathrm{H}\right](7.21), 219$ (100), 91 (9.40), 64 (6.25); Anal. for $\mathrm{C}_{22} \mathrm{H}_{17} \mathrm{ClN}_{2} \mathrm{O}_{3}$ (392.83) calcd; C, 67.26; H, 4.36; Cl, 9.02; N, 7.13; Found: C, 67.13; $\mathrm{H}, 4.35 ; \mathrm{Cl}, 9.08 ; \mathrm{N}, 7.14$.

2-(4-Chlorophenyl)-3-(3,4-dimethoxyphenethyl)quinazolin-4(3H)-one (3c). Compound 3c was prepared from 2-amino- $N$-(3,4-dimethoxyphenethyl)benzamide 1c according to method $\mathrm{A}$, beige cubes; yield (78 $\%$ ); m.p. $150{ }^{\circ} \mathrm{C}$; IR $v_{\max }(\mathrm{KBr}) 1681.50,1614.63 \mathrm{~cm}^{-1} ;{ }^{1} \mathrm{H}-\mathrm{NMR}$ (400 MHz, DMSO- $d_{6}$ ) (ppm): $\delta 7.65(1 \mathrm{H}, \mathrm{d}, J=8.0 \mathrm{~Hz}), 7.34-7.44(5 \mathrm{H}, \mathrm{m}), 7.20(1 \mathrm{H}, \mathrm{t}, J=8.0 \mathrm{~Hz}), 6.83(1 \mathrm{H}, \mathrm{d}, J=8.4 \mathrm{~Hz})$, $6.75(1 \mathrm{H}, \mathrm{s}), 6.68(1 \mathrm{H}, \mathrm{t}, J=8.0 \mathrm{~Hz}), 6.63(1 \mathrm{H}, \mathrm{d}, J=8.4 \mathrm{~Hz}), 3.70,3072$ (each 3H, s, 2(OCH $)$ ), 2.96 $\left(2 \mathrm{H}, \mathrm{t}, J=6.9 \mathrm{~Hz}, \mathrm{CH}_{2}\right), 2.65\left(2 \mathrm{H}, \mathrm{t}, J=7.3 \mathrm{~Hz}, \mathrm{CH}_{2}\right) ;{ }^{13} \mathrm{C}-\mathrm{NMR}: \delta$ 20.0, 35.0, 47.5, 55.8, 112.39, 112.97 (2C), 114.82,115.43, 117.85, 121.03 (2C), 128.00, 128.73 (2C), 129.09 (2C), 131.91, 133.55, 133.86, 140.62, 146.73, 147.80, 149.10, 162.7; MS m/z (\%): $168\left[\mathrm{M}^{+}\right]\left(\mathrm{C}_{24} \mathrm{H}_{21} \mathrm{~N}_{2} \mathrm{O}_{3} \mathrm{Cl}\right)$; Anal. for $\mathrm{C}_{24} \mathrm{H}_{21} \mathrm{ClN}_{2} \mathrm{O}_{3}$ (420.89) calcd; C, 68.49; H, 5.03; Cl, 8.42; N, 6.66; Found: C, 68.51; H, 5.02; Cl, 8.40; $\mathrm{N}, 6.68$.

2-(4-(2-(4-Chlorophenyl)-4-oxoquinazolin-3(4H)-yl)phenyl)acetic Acid (3d). Compound 3d was prepared from 2-(4-(2-aminobenzamido)phenyl)acetic acid 1d according to method A, shining beige needles; yield (65 ${ }^{\mathbf{a}_{0}}$ ); m.p. $213{ }^{\circ} \mathrm{C}$; IR $v_{\max }(\mathrm{KBr}) 3653.46,3109.55,1707.22,1665.45,1620.90 \mathrm{~cm}^{-1}$; ${ }^{1} \mathrm{H}-\mathrm{NMR}\left(400 \mathrm{MHz}, \mathrm{DMSO}-d_{6}\right)(\mathrm{ppm}): \delta 12.32(1 \mathrm{H}, \mathrm{s}, \mathrm{OH}), 8.00(1 \mathrm{H}, \mathrm{d}, J=7.5 \mathrm{~Hz}), 7.71(1 \mathrm{H}, \mathrm{t}$, 
$J=7.5 \mathrm{~Hz}), 7.60-7.66(4 \mathrm{H}, \mathrm{m}), 7.42(1 \mathrm{H}, \mathrm{d}, J=7.5 \mathrm{~Hz}), 7.33(2 \mathrm{H}, \mathrm{d}, J=7.3 \mathrm{~Hz}), 7.28(1 \mathrm{H}, \mathrm{t}, J=7.5 \mathrm{~Hz})$, $7.21(2 \mathrm{H}, \mathrm{d}, J=7.3 \mathrm{~Hz}), 3.34\left(2 \mathrm{H}, \mathrm{s}, \mathrm{CH}_{2}\right)$; ${ }^{13} \mathrm{C}-\mathrm{NMR}: \delta 20.0,115.43,115.87,120.06,126.47(2 \mathrm{C})$, 128.97(2C), 129.77, 130.00(2C), 131.17, 133.17(2C), 133.47,134.43, 139.70, 140.33, 146.86, 162.70, 173.1, 173.4; $\mathrm{MS} m / z(\%): 390\left[\mathrm{M}^{+}\right]\left(\mathrm{C}_{22} \mathrm{H}_{15} \mathrm{~N}_{2} \mathrm{O}_{3} \mathrm{Cl}\right)(3.12), 389\left[\mathrm{M}^{+}-\mathrm{C}_{22} \mathrm{H}_{15} \mathrm{ClN}_{2} \mathrm{O}_{3}\right.$ (390.82) calcd; $\mathrm{C}$, 67.61; H, 3.87; Cl, 9.07; N, 7.17; Found: C, 67.60; H, 3.90; Cl, 9.10; N, 7.18.

\subsection{Antimicrobial Evaluation [30]}

\subsubsection{Antifungal Activity}

Samples of the synthesized molecules were subjected separately in vitro for their antifungal evaluation viz. Aspergillus fumigatus (RCMB 002003), Geotrichum candidum (RCMB 052006) Candida albicans (RCMB 005002) and Syncephalastrum racemosum (RCMB 005003). The cluture of fungi was purified by single spore isolation technique. The antifungal activity was tested by agar well diffusion method according to the following procedure:

Sabourad dextrose agar plates: A homogeneous mixture of glucose-peptone-agar (40:10:15) was sterilized by autoclaving at $121{ }^{\circ} \mathrm{C}$ for $20 \mathrm{~min}$. The sterilized solution $(25 \mathrm{~mL})$ was poured in each sterilized petri dish in a laminar flow hood and left for $20 \mathrm{~min}$. to form the solidified sabourad dextrose agar plate. These plates were inverted and kept at $30^{\circ} \mathrm{C}$ in an incubator to remove the moisture and to check for the contamination.

\subsubsection{Antifungal Assay}

Fungal strain was grown in $5 \mathrm{~mL}$ sabourad dextrose broth (glucose/peptone; 40:10) for 3-4 days to achieve $105 \mathrm{C} \mathrm{FU} / \mathrm{mL}$ cells. The fungal culture $(0.1 \mathrm{~mL})$ was spread out uniformly on the sabourad dextrose agar plates by sterilized triangular folded glass rod. Plates were left for 5-10 min. so that culture is properly adsorbed on the surface of sabourad dextrose agar plates. Small wells of size $(4 \mathrm{~mm} \times 2 \mathrm{~mm})$ were cut into the plates with the help of well cutter and bottom of the wells were sealed with $0.8 \%$ soft agar to prevent the flow of test sample at the bottom of the well. $100 \mu \mathrm{L}$ of the tested samples $(10 \mathrm{mg} / \mathrm{mL})$ were loaded into the wells of the plates. All compounds dissolved in DMSO were loaded as control. The plates were kept for incubation at $30{ }^{\circ} \mathrm{C}$ for $3-4$ days and then the plates were examined for the formation of zone of inhibition. Each inhibition zone was measured three times by caliper to get an average value. The test was performed three times for each fungus. Clotrimaole and itraconazole were used as antifungal standard drugs.

\subsubsection{Antibacterial Activity}

Antibacterial evalution was tested using agar well diffusion method. The activity of tested samples was tested against Staphylococcus aureus (RCMB 000106) and Bacillis subtilis (RCMB 000107), as Gram positive bacteria and Pseudomonas aeruginosa (RCMB 000102) and Escherichia coli (RCMB 000103), as gram negative bacteria. The solution of $5 \mathrm{mg} / \mathrm{mL}$ of each compound in DMSO was used for testing against bacteria. Centrifuged pellets of bacteria from $24 \mathrm{~h}$ old culture containing approximately 104-106 CFU (colony forming unit) per $\mathrm{mL}$ were spread on the surface of nutrient agar (typetone $1 \%$, yeast extract $0.5 \%, \mathrm{NaCl} 0.5 \%$, agar $1000 \mathrm{~mL}$ of distilled water, $\mathrm{pH} 7.0$ ) and 
was autoclaved under $121^{\circ} \mathrm{C}$ for at least $20 \mathrm{~min}$. Wells were created in medium with the help of sterile metallic bores and then cooled down to $45^{\circ} \mathrm{C}$. The activity was determined by measuring the diameter of the inhibition zone (in mm). $100 \mu \mathrm{L}$ of the tested samples $(10 \mathrm{mg} / \mathrm{mL})$ were loaded into the wells of the plates. All compounds were prepared in DMSO, and were loaded as control. The plates were kept for incubation at $37{ }^{\circ} \mathrm{C}$ for $24 \mathrm{~h}$ and then the plates were examined for the formation of zone of inhibition. Each inhibition zone was measured three times by caliper to get an average value. The test was performed three times for each bacteria. Streptomycin was used as an antibacterial standard drug.

\subsubsection{Material and Methods}

To investigate the binding pattern of the Quinazoline derivatives respect to their binding affinity, docking experiments were performed using genetic algorithm approach implemented in the GOLD [33] docking program for conformational search during docking process. PDB ID 1T2W retrieved from Protein Data Bank [34] and the co-crystallized ligand LPXTG peptide complex along with water moelcules were omitted from the targeted protein. Default parameters were set to optimize the docking experiment. All the Ligands were sketched by ChemDraw Ultra and converted into 3D format using OpenEye Babel [35]. Hydrogen's were added and MMFF94 partial charges were assigned to all ligands by MOE [36] prior to minimization. Furthermore, protonation states were corrected using OpenEye Filter [37] program before docking runs. MOE and Poseview [38] were used for the molecular interaction analysis.

\section{Conclusions}

We have successfully prepared quinazolin-4(3H)-one derivatives $\mathbf{2 a}-\mathbf{h}$ and $\mathbf{3 a}-\mathbf{d}$ starting from 2-aminobenzamide derivatives 1 with triethyl orthoformate and $p$-chlorobenzaldehyde, respectively, by one pot synthesis assisted by microwave or classical methods. The simple procedure, mild conditions, high yields and especially environmental friendliness make this protocol very attractive. Compound 3a showed strong inhibitory effect against Gram-negative bacteria (Pseudomonas aeruginosa and Esherichia coli) and Gram-positive bacteria (Staphylococcus aureus, and Bacillus subtilis), in addition to and anti-fungal activity against (Aspergillus fumigatus, Saccharomyces cervevisiae, and Candida albicans).

\section{Acknowledgments}

The authors extend their appreciation to the Deanship of Scientific Research at King Saud University for funding this work through the research group project Number RGP-VPP-007.

\section{Author Contributions}

Yahia N. Mabkhot designed the study, carried out the synthesis. Assem Barakat wrote some research and audit. Munirah S. Al-Har and Fahad D. Aldawsari students did the experiments. Ali Aldalbahi carried out the synthesis and edited the English language. Zaheer-Ul-Haq carried out molecular docking studies. 


\section{Conflicts of Interest}

The authors declare no conflict of interest.

\section{References and Notes}

1. Michael, J.P. Quinoline, quinazoline and acridone alkaloids. Nat. Prod. Rep. 2007, 24, 223-246.

2. Mhaske, S.B.; Argade, N.P. The chemistry of recently isolated naturally occurring quinazolinone alkaloids. Tetrahedron 2006, 62, 9787-9826.

3. Tseng, M.-C.; Yang, H.-Y.; Chu, Y.-H. Total synthesis of asperlicin C, circumdatin F, demethylbenzomalvin $\mathrm{A}$, demethoxycircumdatin $\mathrm{H}$, sclerotigenin, and other fused quinazolinones. Org. Biomol. Chem. 2010, 8, 419-427.

4. Kubicova, L.; Kudelova, P.; Dostal, H.; Waisser, K. Antialgal activity of 3-Phenyl-2H-1, 3-benzoxazine-2,4(3H)-diones and 3-Phenyl-3H-quinazoline-4-ones against Chlorella Vulgaris. Folia Pharm. Univ. Carol. 2000, 25, 81-87.

5. Hanusek, J. Synthesis, kinetics, and biological activity of 2-Phenylquinazoline-4-thiones. Chem. List. 2001, 95, 811-813.

6. William, R.; Detlef, M.; Stephen, E.; Bert, K.; Thomas, S.; Manfred, M. Protein kinase inhibitors of the quinazoline class exert anti-cytomegaloviral activity in vitro and in vivo. Antivir. Res. 2008, 79, 49-61.

7. Chan, J.H.; Hong, J.S.; Kuyper, L.F.; Baccanari, D.P.; Joyner, S.S.; Tansik, R.L.; Boytos, C.M.; Rudolph, S.K. Selective inhibitors of candida albicans dihydrofolate reductase: Activity and selectivity of 5-(Arylthio)-2,4-diaminoquinazolines. J. Med. Chem. 1995, 38, 3608-3616.

8. Castaldo, R.A.; Gump, D.W.; McCormack, J.J. Activity of 2,4-Diaminoquinazoline Compounds against Candida species. J. Antimicrob. Agents Chemother. 1979, 15, 81-86.

9. Harushia, K.; Kiesuke, Y.; Seiko, H.; Shingo, H.; Ryota, K.; Norimitsu, H.; Makoto, M.; Yoshiteru, O. Exploration of a new type of antimalarial compounds based on febrifugine. J. Med. Chem. 2006, 49, 4698-4706.

10. Yen, M.H.; Sheu, J.R.; Peng, I.H.; Lee, Y.M.; Chern, J.W. Pharmacological Activity of DC-015, a Novel Potent and Selective $\alpha 1$-Adrenoceptor Antagonist. J. Pharm. Pharmacol. 1996, 48, 90-95.

11. Yasutaka, T.; Takao, S.; Nobuhisa, W.; Hideyuki, A.; Shigeru, S.; Isao, S. Cyclic GMP phosphodiesterase inhibitors. 2. Requirement of 6-substitution of quinazoline derivatives for potent and selective inhibitory activity. J. Med. Chem. 1994, 37, 2106-2111.

12. Marianne, D.; Fredric, P.; Olivier, C.; Jean-Claude, T.; Jean-Pierre, C.; Yves, B. Synthesis and in vitro cytotoxic evaluation of new derivatives of pyrido[1,2-a]benzimidazolic ring system: The pyrido[1',2':1,2]imidazo[4,5-h]quinazolines. Chem. Pharm. Bull. 2001, 49, 1061-1065.

13. Mani Chandrica, P.; Yakaiah, T.; Raghu Ram Rao, A.; Narsaiah, B.; ChakraReddy, N.; Sridhar, V.; Rao, J.V. Synthesis of novel 4,6-disubstituted quinazoline derivatives, their anti-inflammatory and anti-cancer activity (cytotoxic) against U937 leukemia cell lines. Eur. J. Med. Chem. 2008, 43, 846-852.

14. Hyao, S.; Mvera, M.J.; Strycker, W.; Leipzi, T.; Klup, R.; Hartzler, H. New sedative and hypotensive 3-substituted 2,4(1H,3H)-quinazolinediones. J. Med. Chem. 1965, 8, 807-811. 
15. Cohen, E.; Klarberg, E.; James Vaughan, R., Jr. Quinazolinone sulfonamides. A new class of diuretic agentss. J. Am. Chem. Soc. 1960, 82, 2731-2735.

16. Kenji, M.; Junko, U.; Takashi, S.; Michio, I.; Neil, A.G.; Jin-Chen, Y.; Shusuke, T.; Shoji, O.; Yuji, N. Potent and selective inhibitors of platelet-derived growth factor receptor phosphorylation.

3. Replacement of quinazoline moiety and improvement of metabolic polymorphism of 4-[4-( $N$-substituted (thio)carbamoyl)-1-piperazinyl]-6,7-dimethoxyquinazoline derivatives. J. Med. Chem. 2003, 46, 4910-4925.

17. Joachim, R.; William, P.E.; Stephen, O.; Philip, D.G.C.; Philip, L.W.; Michael, B.; Donald, E.B.; Brian, T.B.; Georgiy, B.; Libing, C.; et al. Quinazolinone derivatives as orally available ghrelin receptor antagonists for the treatment of diabetes and obesity. J. Med. Chem. 2007, 50, 5202-5216.

18. Baba, A.; Kawamura, N.; Makino, H.; Ohta, Y.; Taketomi, S.; Sohda, T. Studies on disease-modifying antirheumatic drugs: Synthesis of novel quinoline and quinazoline derivatives and their anti-inflammatory effect. J. Med. Chem. 1996, 39, 5176-5182.

19. Huang, A.X.; Johanson, M.G.; Li, A.; Liu, J.; Marcus, A.P.; Medina, J.C.; Zhu, L. Cxcr3 antagonists. WO2002083143 A1, 24 October 2002. Chem. Abstr. 2002, 137, 337909.

20. Yarosh, D.B.; Galvin, J.W.; Nay, S.L.; Peña, A.V.; Canning, M.T.; Brown, D.A. Anti-inflammatory activity in skin by biomimetic of Evodia rutaecarpa extract from traditional Chinese medicine. J. Dermatol. Sci. 2006, 42, 13-21.

21. Uddin, R.; Lodhi, M.U.; Ul-Haq, Z. Combined pharmacophore and 3D-QSAR study on a series of Staphylococcus aureus Sortase A inhibitors. Chem. Biol. Drug Des. 2012, 80, 300-314.

22. Mabkhot, Y.N.; Barakat, A.; Al Majid, A.M.A.; Alshahrani, S.; Yousuf S.; Choudhary, M.I. Synthesis, reactions and biological activity of some new bis-heterocyclic ring compounds containing sulphur atom. Chem. Cent. J. 2013, 7, 112-120.

23. Mabkhot, Y.N.; Barakat, A.; Al Majid, A.M.A.; Choudhary, M.I. Synthesis of thieno [2,3-b] thiophene containing bis-heterocycles-novel pharmacophores. Int. J. Mol. Sci. 2013, 14, 5712-5722.

24. Al-Nahary, T.T.; El-Ries, M.A.N.; Mohamed, G.G.; Attia, A.K.; Mabkhot, Y.N.; Harone, M.; Barakat, A. Multiclass analysis on repaglinide, flubendazole, robenidine hydrochloride and danofloxacin drugs. Arab. J. Chem. 2013, 6, 131-144.

25. Mabkhot, Y.N.; Barakat, A.; Al Majid, A.M.A.; Alamary, A.S. A novel and expedient approach to new heterocycles containing thiazole, thiazolo[3,2-a]pyridine, dihydrothiophene, and hydrazonothieno[2,3-b]thiophene moieties. Int. J. Mol. Sci. 2012, 13, 5035-5047.

26. Mabkhot, Y.N.; Barakat, A.; Al Majid, A.M.A.; Alshahrani, S.A. Comprehensive and facile Synthesis of some functionalized bis-heterocyclic compounds containing a thieno[2,3- $b]$ thiophene motif. Int. J. Mol. Sci. 2012, 13, 2263-2275.

27. Mabkhot, Y.N.; Barakat, A.; Al Majid, A.M.A.; Al-Othman, Z.A.; Alamary, S.A. A facileand convenient synthesis of some novel hydrazones, schiff's base and pyrazoles incorporating thieno [2,3-b]thiophenes. Int. J. Mol. Sci. 2011, 12, 7824-7834.

28. Mabkhot, Y.N.; Al Majid, A.M.A.; Barakat, A.; Alshahrani, S.; Siddiqui, Y. 1,1'-(3-Methyl-4phenylthieno[2,3-b]thiophene-2,5-diyl)diethanone as building block in heterocyclic synthesis. Novel synthesis of some pyrazoles, and pyrimidines derivatives. Molecules 2011, 16, 6502-6511. 
29. Mabkhot, Y.N.; Al-Har, M.S.; Barakat, A.; Al-Showiman, S.S. Diversity oriented design of various hydrazides and amides derived from isatoic anhydride and their antimicrobial activity evaluation. Dig. J. Nanomater. Biostruct. 2013, 8, 1345-1355.

30. Mabkhot, Y.N.; Al-Majid, A.M.; Barakat, A.; Al-Showiman, S.S.; Al-Har, M.S.; Radi, S.; Naseer, M.M.; Hadda, T.B. Synthesis and biological evaluation of 2-Aminobenzamide derivatives as antimicrobial agents: Opening/Closing pharmacophore site. Int. J. Mol. Sci. 2014, 15, 5115-5127.

31. Smania, A.; Monache, F.D.; Smania, E.F.A.; Cuneo, R.S. Triterpenes and sterols from ganoderma. australe (Fr.) Pat. (Aphyllophoromycetideae). Int. J. Med. Mushrooms 1999, 1, 325-334.

32. Zong, Y.; Bice, T.W.; Ton-That, H.; Schneewind, O.; Narayana, S.V. Crystal structures of Staphylococcus aureus sortase A and its substrate complex. J. Biol. Chem. 2004, 279, 31383-31389.

33. Cambridge Crytallographic Data Center. GOLD version 5.2; Cambridge Crytallographic Data Center: Cambridge, UK, 2008.

34. RCSD PDB (Protein Data Bank) Homepage. Available online: http://www.rcsb.org/pdb (accessed on 1 January 2014).

35. O’Boyle, N.M.; Banck, M.; James, C.A.; Morley, C.; Vandermeersch, T.; Hutchison, G.R. Open babel: An open chemical toolbox. J. Cheminf. 2011, 3, 1-14.

36. Molecular Operating Environment (MOE), 2012. Chemical Computing Group Inc.: 1010 Sherbooke St. West, Suite \#910, Montreal, QC, H3A 2R7, Canada.

37. OpenEye Scientific Software. OE FILTER version 2.5; OpenEye Scientific Software Inc.: Santa Fe, NM, USA.

38. Stierand, K.; Rarey, M. Poseview molecular interaction patterns at a glance. J. Cheminf. 2010 , 2,50 .

Sample Availability: Samples of the compounds $\mathbf{2 a}-\mathbf{h}$ and $\mathbf{3 a}-\mathbf{d}$ are available from the authors.

(C) 2014 by the authors; licensee MDPI, Basel, Switzerland. This article is an open access article distributed under the terms and conditions of the Creative Commons Attribution license (http://creativecommons.org/licenses/by/3.0/). 